Proceedings

\title{
Sensor Based Gas Leakage Detector System †
}

\author{
Mohammad Monirujjaman Khan \\ Department of Electrical and Computer Engineering, North South University, Bashundhara, Dhaka 1229, \\ Bangladesh; monirujjaman.khan@northsouth.edu; Tel.: +880-1779006296 \\ + Presented at the 7th Electronic Conference on Sensors and Applications, 15-30 November 2020; \\ Available online: https://ecsa-7.sciforum.net/.
}

Published: 15 November 2020

\begin{abstract}
Liquefied Petroleum Gas (LPG) is a main source of fuel especially in urban areas because it is clean compared to firewood and charcoal. Gas leakage is a major problem with industrial sector, residential premises etc. Now a day's home security has been a major issue because of increasing gas leakage. Gas leakage is a great anxiety with ateliers, residential areas and vehicles like CNG, buses, cars which is run by gas-power. One of the preventive methods to stop accident associated with the gas leakage is to install a gas leakage detection kit at vulnerable places. The aim of this paper is to propose and discuss a design of gas leakage detection system that can automatically detect, alert and control gas leakage. This proposed system also includes alerting system to the users. The system is based on a sensor that easily detect a gas leakage.
\end{abstract}

Keywords: LPG (liquefied petroleum gas); gas sensor MQ-6; buzzer (alarm); LED (light)

\section{Introduction}

Gas leakage is serious problem and now a days it is observed in many places like residences, industries, vehicles like CNG, buses, cars, etc. It is noticed that due to gas leakage dangerous accident occurs. The LPG or propane is a flammable mixture of hydrocarbon gases used as fuel in many applications like homes, hostels, industries, automobiles, vehicles because of its desirable properties which include high calorific value, less smoke, less soot, and meager harm to the environment. Liquid petroleum gas (LPG) is highly inflammable and can burn even at some distance from the source of leakage. This energy source is primarily composed of propane and butane which are highly flammable chemical compounds. These gases can catch fire easily. In homes, LPG is used mainly for cooking purpose. When a leak occurs, the leaked gases may lead to explosion. Gas leakage leads to various accidents resulting in both material loss and human injuries. Home fires have been taking place frequently and the threat to human lives and properties is growing in recent years. The risk of explosion, firing, suffocation are based on their physical properties such toxicity, flammability, etc. The number of deaths due to explosion of gas cylinders has been increasing in recent years. Bhopal gas tragedy is an example for accidents due to gas leakage.

The reason for such explosion is due to substandard cylinders, old valves, not regular checking of gas cylinders, worn out regulators and lack of awareness in handling gas cylinders. So the gas leakage should be detected and controlled to protect the people from danger. An odorant such as ethane thiol is added to LPG, so that leaks can be detected easily by most people. However, some people who have a reduced sense of smell may not be able to rely upon this inherent safety mechanism. Gas leakage detector becomes vital and helps to protect people from the dangers of gas leakage. A number of research papers have been published on gas leakage detection techniques [18]. K. Padmapriya et.al, proposed the design of wireless LPG monitoring system. In this paper, the user is alerted about the gas leakage through SMS and the power supply is turned off [6]. Meenakshi Vidya et.al. proposed the leakage detection and real time gas monitoring system. In this system, the 
gas leakage is detected and controlled by means of exhaust fan. The level of LPG in cylinder is also continuously monitored [7]. Selvapriya et.al proposed the system in which the leakage is detected by the gas sensor and produce the results in the audio and visual forms. It provides a design approach on software as well as hardware [8]. In the existing method, different gas sensing technology is used.

In this paper a low cost advanced sensor based gas leakage detector, alert and control system is proposed and discussed. The system is very efficient, user friendly, portable, small in size and cost effective. It will cost only 917 Bangladeshi taka which is equivalent to ten USD.

\section{Method and Materials}

In this paper semiconductor sensors are used to detect LPG gas. MQ6 semiconductor sensor is used. Sensitive material of MQ-6 gas sensor is $\mathrm{SnO}$, which with lower conductivity in clean air. When the target combustible gas exists the sensor conductivity increases along with the rising gas concentration. MQ6 gas sensor has high sensitivity to Propane, Butane and LPG, also response to Natural gas. The sensor could be used to detect different combustible gas, especially Methane; it is with low cost and suitable for different application. The MQ-6 can detect gas concentrations anywhere from 200 to $10,000 \mathrm{ppm}$. The sensor's output is an analog resistance. Figure 1 shows the block diagram of gas leakage detection and alert system.

This system is based on Arduino UNO R3 and MQ-6 gas sensor. When the sensor detects gas in atmosphere, it will give digital output 1 and if gas in not detected the sensor will give digital output 0 . Arduino will receive the sensor output as digital input. If sensor output is high, then the buzzer will start tuning along with the LCD will show that "Gas detected: Yes". If sensor output is low then buzzer will not be tuning, LCD will show that "Gas detected: No". Buzzer is the most commonly consists of a number of switches or sensors connected to control unit that determines if and which button was pushed or a preset time has lapsed, and usually illuminates a light on the appreciate button or control panel, and sounds a warning in the form of a continuous or intermittent buzzing or beeping sound.

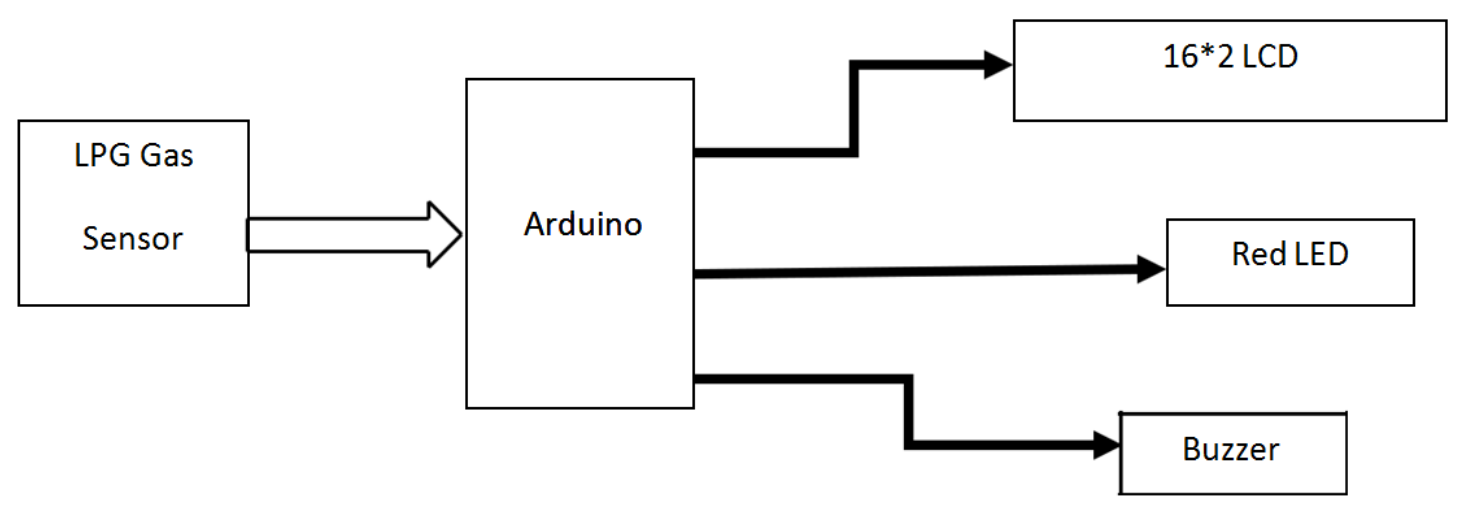

Figure 1. Block diagram of gas leakage detection and alert system.

For the design of sensor based gas leakage detector and alarm system the following hardware components are required. Table 1 listed the list of required hardware opponents, quantity and price in Bangladeshi Taka. The gas detector system is very cheap and it will cost only 917 BD Taka. The device is portable, light weight, user friendly and efficient with multi-functional features. In Figure 2 , some important components that are needed to design the gas leakage detection and alert system are presented. 


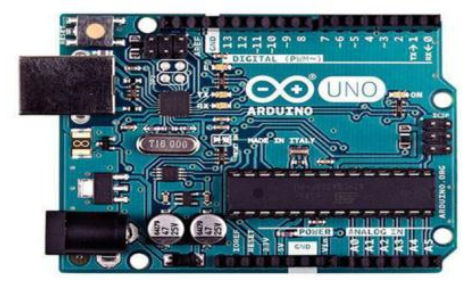

Arduino Uno

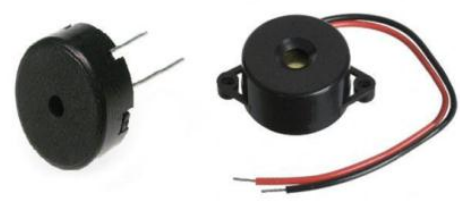

Buzzer

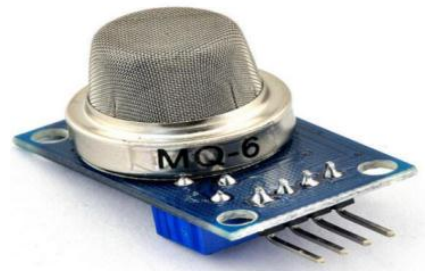

MQ-6 Gas sensor

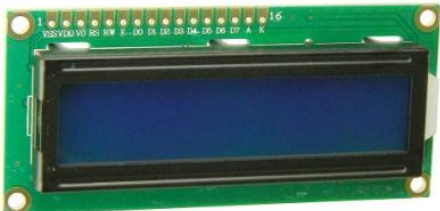

$16^{*} 2 \mathrm{LCD}$

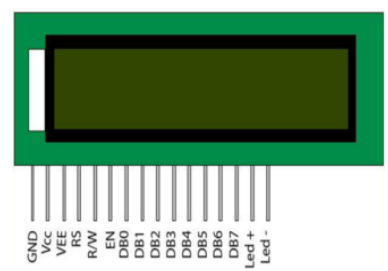

LCD Pin Configuration

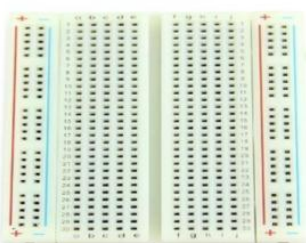

Breadboard

Figure 2. Some important components that are needed to design the gas leakage detection and alert system.

Table 1. List of required hardware opponents, quantity and price in Bangladeshi Taka.

\begin{tabular}{lcc}
\hline \multicolumn{1}{c}{ Equipment } & Quantity & Price (BDT) \\
\hline Arduino Uno R3 & 1 & $420 /-$ \\
MQ-6 LPG gas sensor & 1 & $160 /-$ \\
16*2 LCD & 1 & $124 /-$ \\
Buzzer & 1 & $15 /-$ \\
Male to male/female wire & 40 & $60 /-$ \\
9 V Battery & 1 & $40 /-$ \\
Gas Lighter & 1 & $35 /-$ \\
10 K Variable Resistor & 1 & $8 /-$ \\
Mini Breadboard & 1 & $55 /-$ \\
& & Total Taka-917/- \\
\hline
\end{tabular}

\section{Results and Analysis}

The Proteus Design Suite is a proprietary software tool suite used primarily for electronic design automation. The software is used mainly by electronic design engineers and technicians to create schematics and electronic prints for manufacturing printed circuit boards. Figure 3 shows the circuit diagram that was designed using Proteus libraries. This system is based on Arduino UNO R3 and MQ-6 gas sensor. When the sensor detects gas in atmosphere, it will give digital output 1 and if gas not detected the sensor will give digital output 0 . Arduino will take the sensor output as digital input. If sensor output is high, then the buzzer will start tuning along with the LCD will show that "Gas detected: YES". If sensor output is low then the buzzer will not be tuning, LCD will show that "Gas detected: NO". The detector incorporate MQ-6 sensor (with gas detection range of 300-10,000 ppm) as the LPG gas sensor, PIC16F690 microcontroller as the control unit, LCD for displaying gas concentration, a buzzer as an alarm and a number of LEDs to indicate the gas leakage status. The microcontroller senses the presence of a gas when the voltages signal from the MQ-6 sensor goes beyond a certain level and gives an audiovisual alarm. 


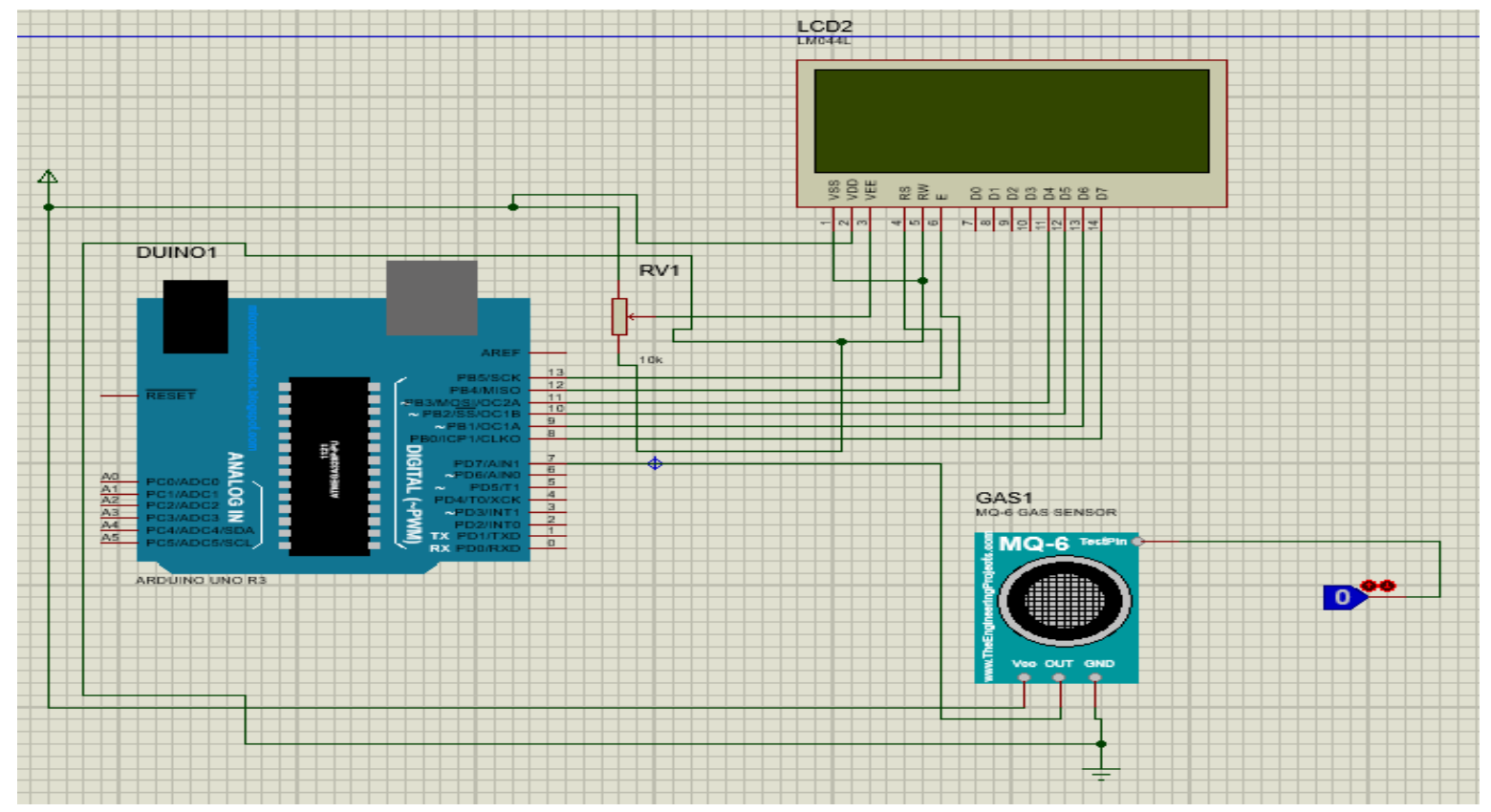

Figure 3. Circuit diagram that was designed using Proteus libraries.

Figure 4 shows the circuit diagram of MQ-6 gas sensor connected with Arduino.

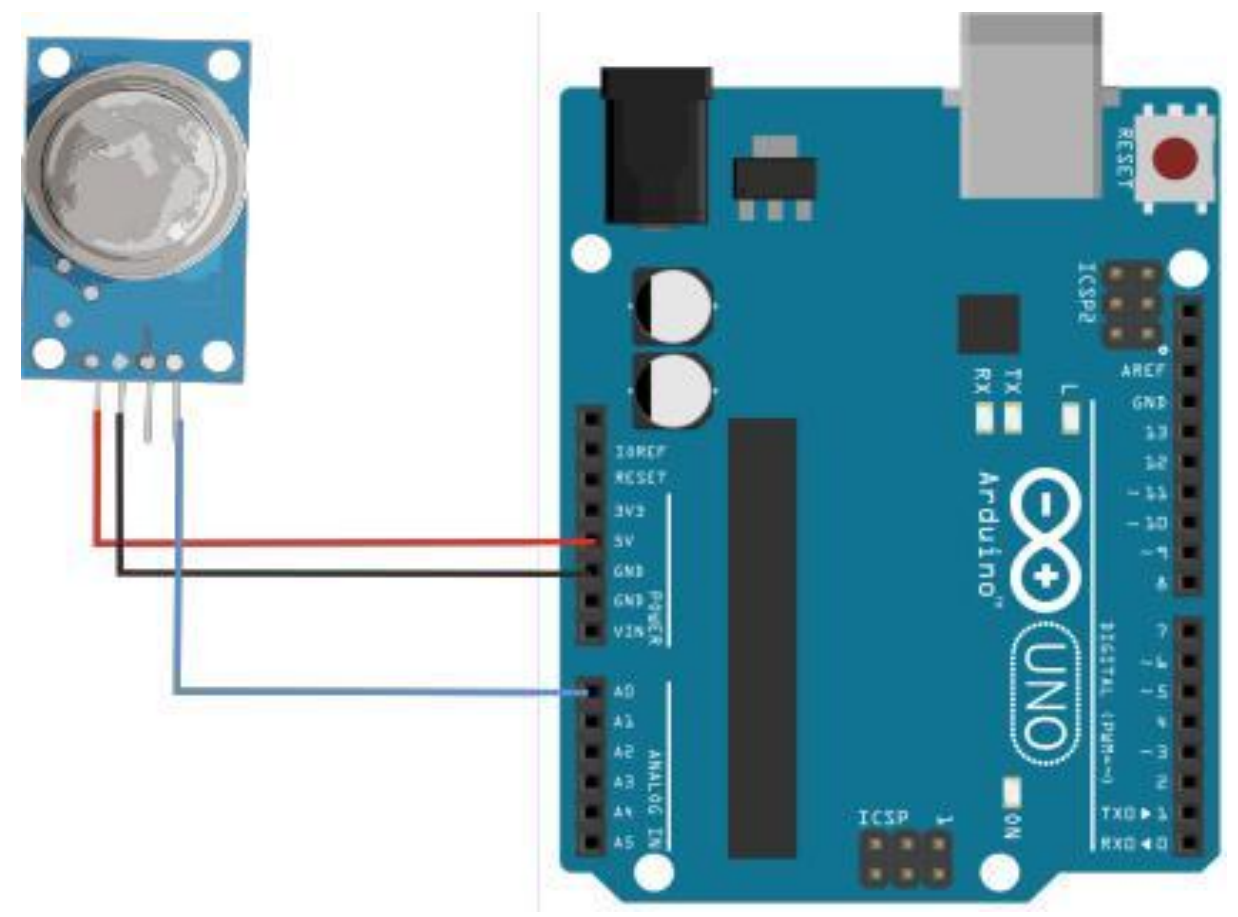

Figure 4. Circuit diagram of MQ-6 gas sensor connected with Arduino.

If the system detects the level of gas in the air that exceeds the safety level it will activate the alarm which includes buzzer to alert the users at home in abnormal condition and to take the necessary action. The most tell-tale sign of a leak is the smell of gas in the home. But in the case of a carbon monoxide leak, there are also particular physical symptoms you may suffer from if there is a leak. The output result of this paper is that the leakage will be detected and stopped within $2 \mathrm{~s}$, after the leakage starts. This system can detect even level of leakage. This is an efficient method for automatically detecting and controlling the gas leakage. Moreover, the fire accidents are also prevented by switching off the power supply. The idea for gas detection and control can be implemented at large scale for various industries. This system can be installed at kitchen, at hostel 
mess, and any other areas. Thus can be helpful in reducing accidents caused due to gas leakage in household as well as in any similar commercial set up. In our country there are 180 million people due to low cost this product is affordable and will save lot of accidents and properties and human lives.

\section{Future Work}

Overall software and hardware parts of the systems have been developed and tested by introducing the small amount of LPG near to gas sensor module. The authors of this paper is currently working to include multi functions with this device. One of the notable future of this system is to add a sub system where wastage of gas and the uses of gas can be monitored using this system. The system is flexible as more number of sensors and relays can be added to it according to the whole LPG supply setup in that premises. Author is adding more software based intelligent functions with this system. This is an automatic gas detection, control and alert system. In future this system will have a features where it can notify the emergency services if any accidents happen. Mobile app and web based app for real time monitoring also will be added. In the user app for this system many smart features will be added. The overall features will make the system more safety for the users. The system will be optimized for the use of many places like car, home, industries and many places. After designing the final prototype with smart multifunctional features, the system will be implemented in real life scenarios as a pilot project. A survey will be done soon before using the system and another one will be done after implementing the system to find out the KPI. Summarizing whole the results, finding and analysis a research article will be written and author has plan to submit in MDPI sensor journal for review. In future paper the features of this final products will be compared with the available gas detector systems presented in other articles.

\section{Conclusions}

Design of a sensor based automatic gas leakage detector with alert and control system have been proposed and discussed in this paper. This is a low-cost, low power, lightweight, portable, safe, user friendly, efficient, multi features and simple system device for detecting Gas. Gas leakage detection will not only provide us the significance in health department but also it will lead to raise our economy because when gas gets leaked it not only contaminates the atmosphere but also wastage of gases will down our economy. The proposed system will cost only USD 10 that is very much affordable even for the poor people. In the open literatures it is noticed that much work has not been done for smart gas detection system. In future more advance features will be integrated with this system which will provide users more safety and relax. The proliferation of handheld devices has led to developments in the field of smart gas sensors, which has considerably widened their scope of application. Need for ensuring safety in workplaces is expected to be the key driving force for the market over the next coming years.

Funding: This research received no external funding.

Acknowledgments: The authors of this paper would like to thank Electrical and Computer Engineering Department at North South University Bangladesh.

Conflicts of Interest: The authors declare no conflict of interest.

\section{References}

1. Mahalingam, A.; Naayagi, R.T.; Mastorakis, N.E. Design and implementation of an economic gas leakage detector. In Recent Researches in Applications of Electrical and Computer Engineering; 2012; pp. 20-24.

2. Attia, H.A.; Halah, Y.A. Electronic Design of Liquefied Petroleum Gas Leakage Monitoring, Alarm, and Protection System Based on Discrete Components. Int. J. Appl. Eng. Res. 2016, 11, 9721-9726.

3. Apeh, S.T.; Erameh, K.B.; Iruansi, U. Design and Development of Kitchen Gas Leakage Detection and Automatic Gas Shut off System. J. Emerg. Trends Eng. Appl. Sci. 2014, 5, 222-228. 
4. Soundarya, T.; Anchitaalagammai, J.V.; Priya, G.D.; Karthickkumar, S.S. C-Leakage: Cylinder LPG Gas Leakage Detection for Home Safety. IOSR J. Electron. Commun. Eng. 2014, 9, 53-58.

5. Shrivastava, A.; Prabhaker, R.; Kumar, R.; Verma, R. GSM based gas leakage detection system. Int. J. Emerg. Trends Electr. Electron. 2013, 3, 42-45.

6. Anurupa, A.; Gunasegaram, M.; Amsaveni, M. Efficient Gas Leakage Detection and Control System using GSM Module. Int. J. Eng. Res. Technol. 2015, 3, 1-4.

7. Meenakshi, A.A.; Meghana, R.B.N.; Krishna, P.R. LPG Gas Leakage Detection and Prevention System. Int. J. Future Revolut. Comput. Sci. Commun. Eng. 2017, 3, 1-4.

8. All Answers Ltd. GSM Based LPG Detection [Internet]. November 2018. Available online: https://ukdiss.com/examples/gsm-based-lpg-detection.php?vref=1 (accessed on 15 October 2020).

Publisher's Note: MDPI stays neutral with regard to jurisdictional claims in published maps and institutional affiliations.

(C) 2020 by the authors. Submitted for possible open access publication under the terms and conditions of the Creative Commons Attribution (CC BY) license (http://creativecommons.org/licenses/by/4.0/). 